\title{
ON THE REPRESENTATION AND APPROXIMATION OF A CLASS OF OPERATOR-VALUED ANALYTIC FUNCTIONS
}

\author{
BY G. D. ALLEN AND F. J. NARCOWICH
}

Communicated by Richard Goldberg, October 17, 1974

1. Main results. Let $H$ be a separable Hilbert space, and $a, b$, real and finite. Suppose that $T(z)$ is, for each $z \notin[a, b]$, a bounded operator-valued analytic function which satisfies the condition

(R) For each $\phi \in H$ and $z \notin[a, b],(T(z) \phi, \phi)$ maps the upper halfplane into itself and the lower half-plane into itself.

We then call $T(z)$ an $R$-operator, and the class of all $R$-operators with the cut $[a, b]$ will be called $W[a, b]$. The class $W[a, b]$ generalizes to operators of the class $R[a, b]$ of $R$-functions, those analytic functions on the cut complex plane which preserve upper and lower half-planes. For any $f(z) \in$ $R[a, b]$, there is a nonnegative number $\alpha$, a real number $\beta$, and a positive measure $\mu$ with support on $[a, b]$, such that

$$
f(z)=\alpha z+\beta+\int_{a}^{b} \frac{d \mu(t)}{t-z}
$$

holds (cf. Stone [5, p. 573]). This representation links Stieltjes transforms (the integral given above) to a mapping condition. The intent of this note is to state a generalization of this result to $R$-operators. Results in this direction have been obtained by J. S. Mac Nerney [3] in connection with the Hermitian moment problem and corresponding continued fractions.

THEOREM 1. If $T(z) \in W[a, b]$, then there is a bounded nonnegative operator $A$, a selfadjoint operator $B$ and a strongly countably additive positive operator-valued measure $\mu$ such that

$$
T(z)=A z+B+\int_{a}^{b} \frac{d \mu(t)}{t-z} .
$$

If, in addition, the $T(z)$ form a compact family of operators, then $\mu(t)$ is uniformly countably additive.

AMS(MOS) subject classifications (1970). Primary 41A20, 47A65; Secondary $81 \mathrm{~A} 10,81 \mathrm{~A} 45$.

Copyright @ 1975, American Mathematical Society 
With this construction of the measure $\mu(t)=\mu([0, t])$, which can be constructed to be left continuous, and assuming $A=B=0$, we obtain several corollaries:

(1) $T(z)$ is called meromorphic if for each $\phi \in H,(T(z) \phi, \phi)$ is a meromorphic function. $T(z)$ is a meromorphic R-operator if and only if the measure $\mu$ is purely atomic.

(2) If $b<\infty$ and $\mu(b) \leqslant I$, the identity operator, there exists a Hilbert space $H_{1} \supseteq H$ and a selfadjoint operator $A$ on $H_{1}$, such that on $H, T(z)=$ $P(A-z I)^{-1}+f(z)(I-\mu(b))$, where $f(z)$ is an $R$-function and $P$ is the orthogonal projection from $\mathrm{H}_{1}$ onto $\mathrm{H}$.

Corollary (2) illustrates the close relation of $R$-operators to resolvents. Also we wish to point out the similarity of our representation theorem to the result in Fillmore [2] that proves the analogous Herglotz representation. To approach the problem from the Naimark extension theory, as would be required in the above situation, one must first have the operator-valued measure constructed in Theorem 1.

Perhaps more important than the representation theorem above is the fact that $R$-operators can be approximated by rational functions which converge uniformly on compact sets off the cut $[a, b]$. The rational approximants to which we refer are the operator-valued analogues of Pade approximants (cf. [6, J. Zinn-Justin]). It is of course no mystery why Pade' approximants are used; in the case of Stieltjes series these approximants converge uniformly on compact sets off the cut $[a, b]$. If the $R$-operator $T(z)$ has the formal power series $\Sigma_{k=0}^{\infty} T_{k} z^{k}$, if $P_{N}(z)$ and $Q_{N}(z)$ are polynomials of degree $N$, and if

$$
P_{N}(z) Q_{N}(z)^{-1}-T(z)=D_{2 N+1} z^{2 N+1}+\cdots,
$$

we say that $P_{N} Q_{N}^{-1}$ is the (right) $[N / N]$ Pade' approximant to $T(z)$. [6, J. Zinn-Justin] shows that the right, left and mixed Pade' approximants are equal if all exist. Precisely then, we state

THEOREM 2. If $T(z) \in W[a, b]$, then for each $N$ there is an $[N / N]$ Padé approximant of mixed type, $T_{N}(z)$, which converges uniformly in the norm topology to $T(z)$ on compact subsets of $C$ bounded away from the cut $[a, b]$.

We remark that the derivation of the Padé approximants comes from a variant of Nuttall's compact formula (cf. [1, Baker ]). 
2. Applications. The above results can be applied to both represent and approximate the "reaction matrix" originally studied by E. P. Wigner. For a modern account of this theory see [4] .

As a second application, consider the Schrödinger time-evolution operator $U(t, z)$ which satisfies

$$
i(d / d t) U(t, z)=\left(H_{0}-z V(t)\right) U(t, z), \quad U(0, z)=I,
$$

where $H_{0}$ is an unperturbed Hamiltonian and $V(t)$ is a bounded uniformly positive definite potential. Although $U(t, z)$ is not an $R$-operator, the operator $T(t, z)=i(I-U)(I+U)^{-1}$ is. If we let $T_{N}$ be the $[N / N]$ Pade' approximant of $T$ and $U_{N}$ that of $U$, then $U_{N}(t, z)=-\left(T_{N}-i\right)\left(T_{N}+i\right)^{-1}$ (cf. [6, J. Zinn-Justin ]). Thus, by Theorem $2, U_{N}$ and $T_{N}$ have roughly the same convergence properties. An essential property of $U(t, z)$ is that it is unitary on the real axis. The Padé approximants preserve this property, whereas the partial sums do not.

\section{REFERENCES}

1. G. Baker, The Padé approximant in theoretical physics, (G. Baker and J. Gammel, Editors), Academic Press, New York, 1970.

2. P. Fillmore, Notes on operator tineory, Van Nostrand Reinhold Math. Studies, No. 30, Van Nostrand Reinhold, New York, 1970. MR 41 \#2414.

3. J. S. Mac Nerney, Hermitian moment sequences, Trans. Amer. Math. Soc. 103 (1962), 45-81. MR 27 \#46.

4. F. Narcowich, The mathematical theory of the R-matrix. II: The R-matrix and its properties, J. Mathematical Phys. 15 (1974), 1635-1642.

5. M. H. Stone, Linear transformations in Hilbert space and their applications to analysis, Amer. Math. Soc., Providence, R. I., 1932.

6. J. Zinn-Justin, Strong interaction dynamics with Padé approximants, Phys. Lett. 1 (1971), 55-102.

DEPARTMENT OF MATHEMATICS, TEXAS A \& M UNIVERSITY, COLLEGE STATION, TEXAS 77843 